\title{
A Comparative Study on Prevalence of Major Reproductive Health Problems of Indigenous and Cross Breed Dairy Cattle in Jimma Zone, South Western Ethiopia
}

\author{
Galana Abaya ${ }^{1}$, Assefa Kebede ${ }^{1}$, Kula Jilo ${ }^{2, ~ * ~}$ \\ ${ }^{1}$ Veterinary Biotechnology, Jimma University, College of Agriculture and Veterinary Medicine, Jimma, Ethiopia \\ ${ }^{2}$ Veterinary Epidemiology, Addis Ababa University, College of Agriculture and Veterinary Medicine, Bishoftu, Ethiopia
}

Email address:

kula.jilo1@gmail.com (K. Jilo)

*Corresponding author

\section{To cite this article:}

Galana Abaya, Assefa Kebede, Kula Jilo. A Comparative Study on Prevalence of Major Reproductive Health Problems of Indigenous and Cross Breed Dairy Cattle in Jimma Zone, South Western Ethiopia. Animal and Veterinary Sciences. Vol. 9, No. 2, 2021, pp. 39-45.

doi: 10.11648/j.avs.20210902.12

Received: December 7, 2020; Accepted: April 21, 2021; Published: April 29, 2021

\begin{abstract}
The objective of the current study was to assess the major reproductive health problems and associated risk factors of dairy cattle in and around Jimma town, South-Western Ethiopia in the current study; out of 343 cows investigated (28\%) were found to be positive for at least one reproductive health problems. The major reproductive health problems identified in the current study were retained fetal membrane (8.75\%), abortion (7\%), dystocia (6.4\%), vaginal prolapsed (2.2\%), still birth $(2.0 \%)$, anestrous $(0.6 \%)$, prolonged calving $(0.6 \%)$ and repeat breeding $(0.6 \%)$. The association was statistically significant $(p<0.05)$. The prevalence of major reproductive health problems were found to be 3.72 times higher in Kersa and 1.43 times higher in Dedo district than Jimma town, while in Agaro and Saka were 0.5 and 0.95 times lower than Jimma town and the difference was statistically significant $(\mathrm{P}=0.029)$. Furthermore, cross breed cows were 0.55 times less likely prone to reproductive health problems than local breeds and the difference statistically significant $(\mathrm{P}=0.01)$. The multi-parous cows were 1.4 times more vulnerable to reproductive health problems than primiparous $(\mathrm{P}=0.007)$. Body condition score was also significantly contributing to the presence of reproductive health problems $(\mathrm{P}=.011)$. Current study revealed that major reproductive health problems are responsible for the low reproductive performance of dairy cows in the study area. Therefore, creating awareness among farm owners and animal attendants concerning reproductive health problems and their prevention mechanisms, as well as further study on identifying the specific causative agents were recommended of reproductive health problems should be conducted.
\end{abstract}

Keywords: Cross Breeds, Dairy Cow, Ethiopia, Indigenous, Jimma Zone, Reproductive Health Problem

\section{Introduction}

Ethiopian economy is highly dependent on agriculture which contributed about $47 \%$ of the GDP in the country. Out of this, livestock production accounts for approximately $30 \%$ of the total agricultural GDP and $16 \%$ of the national foreign currency earnings [1] Ethiopia has a solid and long tradition for livestock keeping history. According to the Central Statistical Agency report, the livestock population of the country is estimated at 45.13 million heads of cattle, 24.2 million heads of sheep, 22.6 million of goats, 0.99 million heads of camels, 8.73 million equines and 48.89 million chickens, which have a significant contribution to the national economy. When compared to the livestock population in the country, cattle population ranked second next to chickens and from the total cattle population, $45.13 \%$ are males and $54.87 \%$ females. This indicates the importance of cattle to the country's economy [1].

Despite the huge number of cattle and their economic importance, the productivity is low due to the constraints such as diseases of different origin, low quality and quantity of nutrition, poor management and poor performance of indigenous breeds. These constraints result in poor reproductive of dairy cattle [2] Reproductive disorders have 
been found to be a major reason for decreased reproductive efficiency in cattle and consequently, reproductive efficiency is the major determinant of life time productivity of cows [3]. In the last few decades, as the major epidemic disease were under control, emphasis has increasingly shifted to economically important diseases to the dairy producers [4]. In order to breed regularly, the cow has to have normally functioning reproductive system such as functional ovaries, display estrous behavior, mate, conceive, sustain the embryo through gestation, calve and resume estrous cyclicity and restore uterine function after calving. Each of these aspects of reproductive function can be affected by management, disease and the genetic make-up of the animal. When the function of the reproductive system is impaired, cows fail to produce a calf regularly [5].

Among the major reproductive problems that have direct impact on reproductive performance of dairy cows are abortion, dystocia, retained fetal membrane (RFM), pyometra, metritis, prolapsed (uterine and vaginal), anoestrous and repeat breeding. They are classified as before gestation (anoestrous and repeat breeding), during gestation (abortion, vaginal prolapsed and dystocia) and after gestation (retained fetal membrane and uterine prolapsed [3]. Upon closer examination of reproductive processes in the dairy cattle, the post-partum period is the most varied and vulnerable to problems which incidentally coincide with the peak of milk production, uterine involution and resumption of ovarian activity, conception and greater risk to infection [6]. These results is in considerable economic losses to the dairy industry due to slower uterine involution, reduced reproductive rate, prolonged inter-conception and calving interval, negative effect on fertility, increased cost of medication, drop in milk production, reduced calf crop and early depreciation of potentially useful cows $[7,8]$.

It is very difficult to diagnose those problems by one particular disorder or symptom because there is an interrelation between predisposing factors such as management at calving, hygiene and parity, stage of gestation, nutrition and environment $[8,9]$. Even though there are some studies at other places, there is information gap for Jimma town and its surroundings, thus this study was initiated with the objective of; assessing the prevalence of major reproductive health problems of indigenous and cross breed dairy cattle and identifying the possible risk factors, which play vital roles in causing of such reproductive health problems in dairy farms in the area.

\section{Materials and Methods}

\subsection{The Study Area}

The study was conducted from December 2015 to April 2016 in and around Jimma town. Those surrounding districts were Kersa, Agaro, Dedo and Saka. Jimma town is located at $357 \mathrm{~km}$ southwest of Addis Ababa at about $70^{\circ} 33$, ,N latitude and $36^{\circ} 57^{\circ \mathrm{E}} \mathrm{E}$ longitude, at an altitude of $1710 \mathrm{~m}$ above sea level. The area is characterized by humid climate of heavy rainfall, ranges from 1200 to $2000 \mathrm{~mm}$ per year. About $70 \%$ of the total annual rainfall is received during rainy season, which lasts in early September. The mean maximum and minimum temperature ranges between 26.8 and $11.4^{\circ} \mathrm{C}$ by relative humidity 91.4 and $39.92 \%$, respectively [10]. Total life stock population of Jimma Zone was estimated to be 2.02 million cattle, 288411 goats, 942908 sheep, 152434 equines, 1139735 poultry and 418831 beehives [11].

\subsection{The study Animals and Husbandry Practice}

The study animals were indigenous and crossbred dairy cows, managed under intensive and extensive farming systems. All cows kept for dairy purpose were included in this study.

\subsection{Sampling Strategy}

Jimma City and Kersa, Agaro, Dedo and Saka districts were purposively selected for their large and small scale dairy holder capacity, respectively. A total of 38 dairy owners were randomly chosen from 72 small-scale dairy farmers, registered at Jimma town Bureau of Urban Agriculture Development. One dairy enterprises and one medium size dairy farm of Jimma University were purposively included in this study.

\subsection{Sample Size}

The desired sample size for this study was calculated using the formula stated by Thrusfield with $95 \%$ confidence interval [12]. From previous studies, the average overall prevalence of reproductive health problem in the study area for last five years was found to be $33.59 \%$ and this is taken as expected prevalence (Gashaw et al., 2011). The proposed sample size was calculated by using the following formula: Where; $\mathrm{n}=$ required sample size $\mathrm{Z}=1.96 \mathrm{Pexp}=33.59 \% \mathrm{~d}=$ Desired absolute precision at $95 \%$ and confidence interval $=5 \%$. Thus, 343 dairy cows (211 cross breed and 132 local) were included in this study and 30 pregnant cows were purposively selected from both Jimma University Kito Furdissa General Development Enterprise Dairy Farm and College of Agriculture and Veterinary Medicine Dairy Farm for follow up purpose.

\subsection{Study Design}

A combination of cross-sectional, observational, semistructured questionnaire survey and regular follow up on targeted cows were implemented.

\subsection{Questionnaire Survey}

The objective of this study was explained to workers as well as animal owners before the start of the interview in which oral consent was fixed. Then questions were asked about major reproductive disorders like abortion, dystocia, retained fetal membrane, clinical endometritis, repeat breeder, management systems, parity and body condition. Study animals were grouped according to Nicholson and Butterworth [13]. Then animals were grouped in to the following categories: animals with major reproductive 
disorders and those without these problems.

\subsection{Regular Follow up}

Thirty pregnant cows that were expected to give birth within the study period were selected randomly from Jimma University Kito Furdissa General Development Enterprise (JUKGDEF) and College of Agriculture and Veterinary Medicine (JUCAVM) dairy farm. A regular farms visit of once per week was carried out to collect data on major reproductive health problems of dairy cows. The study animals were identified by their tag number/ID, parity and followed up the rest of their gestation time until delivery and some post-delivery days, by referring their individual case book at the time of delivery. After delivery, cows were examined for the presence of retained fetal membrane left hanging, behind the vulva in the first $24 \mathrm{~h}$ and abnormal discharge occurred in the following post-partum days if any.

\subsection{Data Quality Assurance}

To assure data consistence, two days training was given for dairy farm owners and animal attendants. It was also assured through utilizing reviews data collecting methods which was important for supplementing, complementing and verifying data from each other. Additionally, the questionnaire was pretested to assure its validity and reliability. All points on discussion was also consciously transcribed, coded and printed out in template paper. Cross checking was done by looking for variation among the recorded, transcribed and coded data.

\subsection{Data Management and Analysis}

The collected information was initially entered into a Microsoft excel spread sheet before analysis. Data were analyzed using Statistical Package for the Social Sciences version 20 (SPSS Inc, USA). The chi-square test was used to test for the association among categorical variables. All variables were considered significant at $p \leq 0.05$. Furthermore, descriptive data were summarized using descriptive statistics.

\section{Result}

Socioeconomic characteristics of the respondents and animal husbandry

The respondents constitute $69 \%$ male and $31 \%$ female, who were between 29 to 69 years old. Educational level of respondents was $34.5 \%$ illiterate, $53.4 \%$ read and write, $6.9 \%$ certificate holder and 5.2\% diploma holder and above. However, there were no female individuals having diploma and above. The majority of female respondents were illiterates and the marital statuses of the settlers were $93.1 \%$ married, $1.7 \%$ single and $5.2 \%$ widowed. The residences of the respondents were $41.4 \%$ urban and $58.6 \%$ rural. About $6.9 \%$ were formally employed, $58.6 \%$ farmers, $31 \%$ dairy producer and $3.4 \%$ retired, $58.6 \%$ of the total respondents have no income, $6.9 \%$ have an income less than 500ETB per month, $6.9 \%$ have 500 to 1000 ETB per month while $27.6 \%$ of the total respondents have 1000 ETB above per month.
Out of the total respondents, $51.7 \%$ have less than five family sizes while $48.3 \%$ have above six. Also, $13.8 \%$ uses artificial insemination (AI), 55.2\% uses natural, 27.6\% use both AI and natural and $3.4 \%$ uses communal bull. Concerning animal feeds, $62.1 \%$ get feeds only one per day, $29.35 \%$ two times per day, $6.95 \%$ three times per day while $1.7 \%$ four times per day which depends on the level of income for the respondents. The types of feed given to the dairy cows were hay, straw and concentrate. The ration given was as follow: only hay $(67.25 \%)$, both straw and hay (3.4\%), hay and concentrate $(24.1 \%)$ and $5.2 \%$ all forms of feeds (Water per day also varies according to management system and $34.5 \%$ of the total respondents ${ }^{\text {ee }}$ gave their animals adlibitum); $55.2 \%$ gave two times per day while $10.3 \%$ gave three times per day.

The table below shows $84.5 \%$ of the total respondents practice culling of cows due to, the presence of different reproductive health problems and poor reproductive performance of dairy cows (Table 1). In the current study, out of 343 dairy cows examined, $96(28 \%)$ of them were having at least one or more reproductive health problems (Table 2). Some of the major reproductive health problems identified were: retained fetal membrane, abortion, dystocia, vaginal prolapse, stillbirth, repeat breeding, anestrous and prolonged calving (Table 3). However; retained fetal membrane, abortion and dystocia were the major contributor for dairy cow reproductive health problems, accounting for $8.75,7$ and $6.4 \%$, respectively. Other reproductive health problems were observed with lower prevalence which include vaginal prolapse $(2.2 \%)$ and still birth $(2 \%)$ and anestrous, repeated breeding and prolonged calving interval were $0.6 \%$ each (Table 3 ).

Table 1. Culling practice and reasons of culling.

\begin{tabular}{ll}
\hline Reason of culling & Percentage \\
\hline Age & $29.3 \%$ \\
Age, fertility and health & $8.6 \%$ \\
Age, fertility, health and performance & $5.2 \%$ \\
Age, fertility and performance & $5.2 \%$ \\
Age and health & $24.1 \%$ \\
Age, health and feed scarcity & $1.7 \%$ \\
Age, health, performance & $5.2 \%$ \\
Age and performance & $3.4 \%$ \\
Age and feed scarcity & $1.7 \%$ \\
Total & $84.5 \%$ \\
\hline
\end{tabular}

There was a strong association between reproductive health problems and different risk factors (breeds, parity, body condition, and management systems), and the difference was statistically significant. For example, major reproductive health problems were significantly decreased from cross breed cows to local with the prevalence of 14.3 and $13.7 \%$, respectively which was statistically significant at $\mathrm{p}=0.025$. Multiparous cows $(21.6 \%)$ also showed more prevalence of reproductive health problems compared to primiparous cows $(6.4 \%)$ with parity, significantly contributing to the presence or absence of these diseases ( $p=$ 0.007). The effect of body condition on the prevalence of the major reproductive health problems showed a decrease trend from, animals with good body condition $(8.75 \%)$ to animals 
with poor body condition (7.87). Generally, body condition major reproductive health problems (Table 4). had significant effect $(\mathrm{p}=0.011)$ on the prevalence of the

Table 2. Reproductive Health Problems in and around Jimma town under different study approaches.

\begin{tabular}{llll}
\hline Method of study & No. of cows examined & No. of cows affected & Prevalence (\%) \\
\hline Questionnaire survey & 313 & 88 & $25.66 \%$ \\
Regular follow up & 30 & 8 & $2.33 \%$ \\
Total & 343 & 96 & $28 \%$ \\
\hline
\end{tabular}

Table 3. Relative occurrence of Major Reproductive Health problems in and around Jimma.

\begin{tabular}{llll}
\hline Reproductive health problems & Questionnaire survey no.(\%) & Regular follow up no.(\%) & Total \\
\hline Abortion & $23(7.3 \%)$ & $1(3.3 \%)$ & $24(7 \%)$ \\
Anestrous & $2(.6 \%)$ & 0 & $2(0.6 \%)$ \\
Dystocia & $20(6.4 \%)$ & $2(6.6 \%)$ & $22(6.4 \%)$ \\
Vaginal prolapsed & $7(2.2 \%)$ & 0 & $7(2.2 \%)$ \\
Prolonged calving & $2(.6 \%)$ & 0 & $2(0.6 \%)$ \\
Repeat breeding & $2(.6 \%)$ & 0 & $2(0.6 \%)$ \\
Retained fetal membrane & $26(8.3 \%)$ & $4(13.33 \%)$ & $30(8.75 \%)$ \\
Still birth & $6(1.9 \%)$ & $1(3.3 \%)$ & $7(2 \%)$ \\
Total & $88(28.12 \%)$ & $8(26.67 \%)$ & $96(28 \%)$ \\
\hline
\end{tabular}

There was a difference in the prevalence of reproductive health problems, among the different districts (Woredas) which was statistically significant (Table 5). In general, the prevalence of reproductive health problems in Kersa and Dedo districts were $3.72(\mathrm{CI}=1.145$ to 12.0932$)$ and $1.43(\mathrm{CI}=$ 0.5493 to 3.7277 ) times higher than Jimma town; while Agaro and Sakawere were $0.5(\mathrm{CI}=0.1681$ to 1.5247$)$ and $0.95(\mathrm{CI}=$ 0.3302 to 2.7285 ) times lower than Jimma town, the difference was statistically significant $(\mathrm{P}=0.029)$. Furthermore, cross breed cows were $0.55(\mathrm{CI}=3389$ to 0.8827$)$ times, more likely prone to reproductive health problems than local breed and the difference was statistically significant $(\mathrm{P}=0.01)$. Multiparous cows were $1.4(\mathrm{CI}=0.8151$ to 2.4431$)$ times vulnerable to reproductive health problems than primiparous, which was statistically significant $(\mathrm{P}=0.007)$. Poor and medium body condition cows were $0.92(\mathrm{CI}=0.4810$ to 1.7493$)$ and 0.49 $(\mathrm{CI}=0.2741$ to 0.8588$)$ times, more likely to be affected by reproductive health problems than cows with good body condition, respectively and the difference was statistically significant $(\mathrm{P}=.011)$ (Table 6).

Table 4. Effect of different risk factors on the prevalence of major reproductive health problem.

\begin{tabular}{lllll}
\hline Risk Factors & Total no. of cows examined & Total no. of cows affected (\%) & $\mathbf{X}^{2}$ & P value \\
\hline Breed & & & 49.6 & 0.025 \\
Local & 132 & $47(13.7 \%)$ & 49.6 & 0.025 \\
Cross & 211 & $49(14.3 \%)$ & & \\
Total & 343 & $96(28 \%)$ & 21.09 & 0.007 \\
Parity & & & & \\
Primiparous & 95 & $22(6.4 \%)$ & 31.75 \\
Multiparous & 248 & $74(21.6 \%)$ & \\
Total & 343 & $96(28 \%)$ & & \\
Body condition & & & \\
Poor & 79 & $27(7.87 \%)$ & \\
Medium & 181 & $39(11.37 \%)$ & \\
Good & 83 & $30(8.75 \%)$ & \\
Total & 343 & $96(28 \%)$ & \\
Management system & & & & \\
Intensive & 211 & $49(14.3 \%)$ & \\
Extensive & 132 & $47(13.7 \%)$ & \\
Total & 343 & $96(28 \%)$ & \\
\hline
\end{tabular}

The major reproductive health problems identified under the follow up study of 30 pregnant dairy cows with their frequency was: RFM (13.33\%), dystocia (6.67\%), and abortion/or still birth $(3.33 \%)$, respectively (Figure 1$)$. From the follow up study, major reproductive health problems were relatively higher in JUCAVM than Kito Furdissa General Development
Enterprise Dairy Farm with the prevalence of 40 and 24\%, respectively, which was not statistically significant $(\mathrm{P}=0.673)$. Primiparous cows were less vulnerable to major reproductive health problems than Multiparuos cows with the prevalence of 12.5 and $31.8 \%$, respectively and the association was not statistically significant $(\mathrm{P}=0.780)$. On the other hand, cows 
with good body condition score were more prone to the problems than cows with medium body condition score with prevalence of 33.33 and $28.57 \%$, respectively, though not statistically significant $(\mathrm{p}=0.811)$ (Table 7).

Table 5. Relative occurrence of major reproductive health problems in different Districts (Woredas).

\begin{tabular}{|c|c|c|c|c|c|}
\hline Woreda & No Cows examined & No. cows affected & Prevalence (\%) & $x^{2}$ & P value \\
\hline Jimma town & 267 & 73 & $21.3 \%$ & & \\
\hline Agaro & 25 & 4 & $1.2 \%$ & & \\
\hline Kersa & 12 & 7 & $2 \%$ & 49.57 & 0.025 \\
\hline Dedo & 20 & 7 & $2 \%$ & & \\
\hline
\end{tabular}

Table 6. Effect o different risk factors in reproductive health problems of dairy cows using multivariate logistic regression.

\begin{tabular}{|c|c|c|c|c|c|}
\hline Variables & Total no. examined & Affected cow with RPS & Odds Ratio & P-value & $95 \% \mathrm{CI}$ \\
\hline \multicolumn{6}{|l|}{ District } \\
\hline Jimma & 267 & 73 & Ref. & Ref. & Ref. \\
\hline Agaro & 25 & 4 & 0.5 & 0.226 & $0.1681-1.5247$ \\
\hline Kersa & 12 & 7 & 3.72 & 0.029 & $1.145-12.0932$ \\
\hline Saka & 19 & 5 & 0.95 & 0.92 & $0.3302-2.7285$ \\
\hline Dedo & 20 & 7 & 1.43 & 0.73 & $0.5493-3.7277$ \\
\hline Local & 132 & 47 & Ref. & Ref. & Ref. \\
\hline Cross & 211 & 49 & 0.55 & 0.01 & $0.3389-0.8827$ \\
\hline \multicolumn{6}{|l|}{ Parity } \\
\hline Primiparous & 95 & 22 & Ref. & Ref. & Ref. \\
\hline Maltiparous & 248 & 74 & 1.4 & 0.22 & $0.8151-2.4431$ \\
\hline \multicolumn{6}{|c|}{ Management system } \\
\hline Extensive & 132 & 47 & Ref. & Ref. & Ref. \\
\hline \multicolumn{6}{|l|}{ BCS } \\
\hline Poor & 79 & 27 & 0.92 & 0.79 & $0.4810-1.7493$ \\
\hline Medium & 181 & 39 & 0.49 & 0.01 & $0.2741-0.8588$ \\
\hline Good & 83 & 30 & Ref. & Ref. & Ref. \\
\hline
\end{tabular}

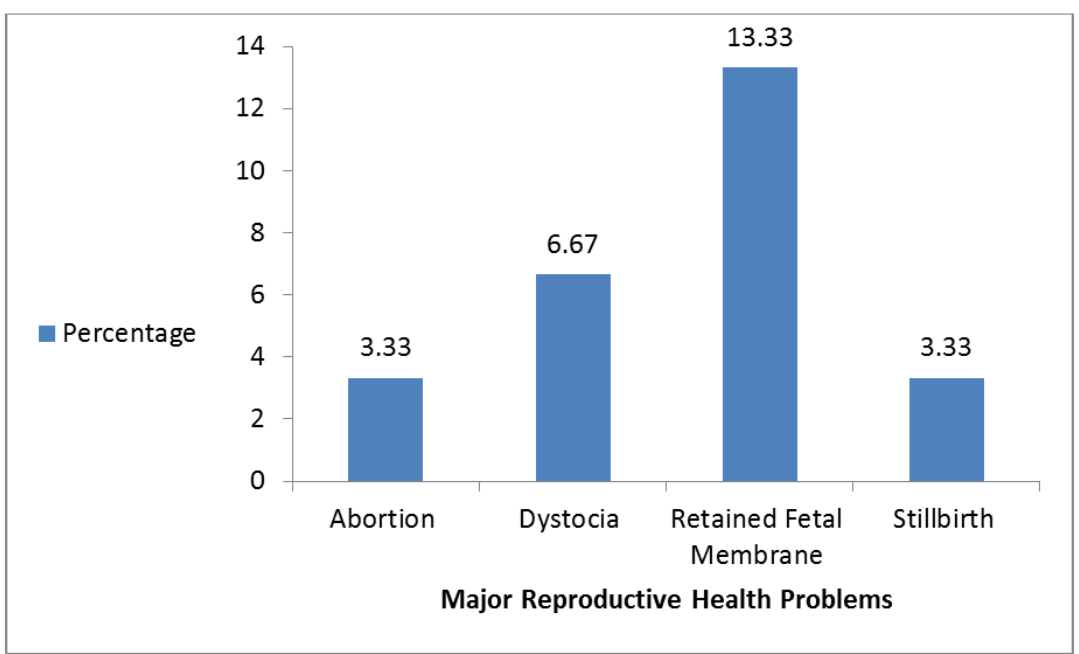

Figure 1. Major Reproductive health problems encountered and their frequency in percentage in JUCAVM and JUKGDEF during Regular follow up ( $\mathrm{n}=30$ ).

Table 7. Result of major reproductive health problems of dairy cows in Jimma University General Development enterprise and College of Agriculture and Veterinary Medicine Dairy farms.

\begin{tabular}{|c|c|c|c|c|c|c|}
\hline Factors & & Total examined & Total affected & Prevalence (\%) & $\mathbf{X}^{2}$ & P_Value \\
\hline \multirow[t]{2}{*}{ Farm } & JUKFGDEF & 25 & 6 & $24 \%$ & 2.35 & 0.673 \\
\hline & JUCAVM & 5 & 2 & $40 \%$ & & \\
\hline \multirow[t]{2}{*}{ Parity } & Primiparious & 8 & 1 & $12.5 \%$ & 1.76 & 0.780 \\
\hline & Multipariuos & 22 & 7 & $31.8 \%$ & & \\
\hline \multirow[t]{3}{*}{ BCS } & Good & 6 & 2 & $33.33 \%$ & 4.48 & 0.811 \\
\hline & Medium & 21 & 6 & $28.57 \%$ & & \\
\hline & Poor & 3 & 0 & & & \\
\hline
\end{tabular}




\section{Discussion}

In this study, $28 \%$ of dairy cattle were affected by at least one or more reproductive health problems. This is in close agreement with the report of Bitew and Prasad [14], who reports a prevalence of $26.5 \%$, but lowers than Gashaw and colleagues [15] in south western Ethiopia and Hadush and colleagues [16] in central Ethiopia, who reported 33.59 and $44.3 \%$, respectively. This difference might be due to the difference in breed, nutrition availability, veterinary service, husbandry practice and environmental factors. The prevalence abortion was 7\% less than Bitew and Prasad [14], findings from Bedelle, who reported 13.9\%. However, these findings agree with those of Getachew [15] and Nibret and Temesgen and Tegegn [17], who reported a prevalence of 5.3 and $5.9 \%$ in Debrezeit and Bako, respectively. This variation might be due to variation in husbandry practices [15] According to Roberts if the incidence rate of abortion is more than 2 to $5 \%$, it should be considered as serious problem and efforts must be made to determine its cause so that, proper control methods might be instituted [6].

The prevalence rate of retained fetal membrane was found to be $8.75 \%$. This was in agreement with the findings of Bitew and Prasad [14], who reported 8.6\%; however, this was lower than the findings of Mamo [18] and Gashaw et al, [15] who reported 14.28 and $19.2 \%$, respectively. The variation in the prevalence of RFM may be due to variation in nutritional status and management system such as lack of exercise, poor sanitation, breed, animal service delivery, age and parity. As the age of the animal increase the ability of the cow to expel fetal material decrease. According to Gashaw et al. [15], dystocia and abortion are important predisposing factors for RFM. Hence, the present increase in RFM rate might be associated with the presence of dystocia and abortion which accounts 6.4 and $7 \%$, respectively.

The prevalence of dystocia was $6.4 \%$. These findings are in agreement with Adane et al [19] from in and around Hosanna, Bitew and Prasad [14], in and around Bedelle, Temesgen and Tegegn [17] in Bako agricultural research and Mamo [18] in small holder dairy cows and in and around Debrezeit who have reported 5.9, 6.64, 6.7 and 5.79\%, respectively. However, the current prevalence was lower than the prevalence of $7.75 \%$ as reported by Tesfaye and Shamble [20] and higher than those of Getachew and Nibret [21] in Debrezeit and Gashaw et al. [15] in Jimma, who have reported 3.3 and $3.8 \%$, respectively. This variation might be due to variation in age and parity of the dam as well as breed of the sire, management system and over feeding. It might be associated with miss matching of the size of birth canal of dam and their fetuses from improved sires [22]. Moreover, the prevalence rate of vaginal prolapse was found to be $2.2 \%$. This was nearly similar with the report of Adane et al [19] from in and around Hosanna who reported $2.05 \%$. However, this finding was lower than Kidusan [23] who reported 5.2\% from Wukro, higher than Molalegn and Shiv [24], who reported a prevalence of $0.66 \%$ from Bedelle. This variation might be due to management system such as feeding and sanitation and environmental factors.

In other side, the prevalence of repeated breeding was $0.6 \%$. This was in agreement with the findings of Gashaw et al. [15] in Jimma, who reported $1.3 \%$ but lower than that of Adane et al. [19], Benti and Zewdie [25], in Borena who had reported a prevalence of 13.08 and $10.3 \%$, respectively. This variation might be associated with a number of factors such as sub-fertile bulls, endocrine imbalance, malnutrition, reproductive tract infections and poor management practices such as wrong time of insemination techniques. In addition to these, communal use of bull from natural services and use of semen from unknown sources might contribute to this particular case. The prevalence of anestrous obtained in this study was $0.6 \%$. This finding was in line with Gashaw et al. [15] and Temesgen and Tegegn [17] who have reported 0.3 and $0.76 \%$, respectively but, the present result was lower than the findings of Benti and Zewdie [25] and Adane et al [19] who had reported a prevalence of 10.3 and $10.25 \%$, respectively. This variation might be due to age, nutrition, uterine infection, breed, and management system differences. Finally, the prevalence rate of still birth in the current study was $2 \%$ which was in agreement with Temesgen and Tegegn who reported $4.8 \%$ Bako research center [17].

\section{Conclusions and Recommendations}

The final goal in each farm should be, to shorten calving interval of cow, decrease the number of services per conception and thereby increase farm production. Reproductive health disorders such as retained fetal membranes, abortion, dystocia, vaginal prolapsed, repeat breeding, anestrous, prolonged calving and still birth affect the reproductive performance of dairy cows, the number of potential replacement needed to maintain a constant dairy cow's size and longevity of the cow in the farm. This study found out that, reproductive disorders most of the time occurs as a complex rather than appearing as a single abnormality. This also revealed a high prevalence of reproductive health problems, out of which RFM, abortion and dystocia, were the most prevalent problems of dairy cows in and around Jimma town. High prevalence of such interrelated problems require further study, use to identify the most important one in designing control strategy and community awareness on its early control and prevention activities, in the study area. Parity, body condition, breeds and management system are some of risk factors identified for the occurrence of reproductive health problems in the study area. Based on the foregone, the following recommendations are forwarded:

1) Routine and periodical examination of cows should be practiced during postpartum and pre-partum period; since most cows acquire reproductive problem during this periods.

2) Awareness creation among farm owners and animal attendants regarding reproductive health problems and their prevention mechanisms should be known. 
3) Proper feeding is very important to control the reproductive problems as found in this study such that, cows having good and poor body conditions will be affected to some extent, by reproductive health problems.

4) Further study should be conducted on identifying the specific causative agents of reproductive health problems.

\section{Conflict of Interests}

The authors have not declared any conflict of interests.

\section{References}

[1] Central Statistical Authority (CSA) (2012). Agricultural sample survey. Volume II: Report on livestock and livestock characteristics. Addis Ababa, Ethiopia.

[2] Shiferaw Y, Tenhagen BA, Bekana M, Kassa T (2005). Reproductive disorders of cross bred dairy cows in the central highlands of Ethiopia and their effect on reproductive performance. Trop. Anim. Health Prod. 37 (5): 427-441.

[3] Lobago F, Bekana M, Gustafsson H, Kindahl H (2006). Reproductive performances of dairy cows in $\mathrm{s}$ mallholder production system in Selalle, Central Ethiopia. Trop. Anim. Health Prod. 38 (4): 333-342.

[4] Hurrissa B, Eshetu J (2002). Challenges and opportunities of livestock marketing in Ethiopia. In Proceedings of the 10th annual conference of the Ethiopian Society of Animal Production (ESAP). Addis Ababa, Ethiopia. pp. 1-13.

[5] Arthur GH, Noakes DE, Pearson H, Perkinson TJ (1996). Veterinary reproduction and obstetrics. Theriogenology 4th ed. Bailliere Tindal. Great Britain. pp. 291-301. Available at: https://www.abebooks.com/servlet/BookDetailsPL?bi=901341 $3207 \&$ sarchurl $=\mathrm{kn} \% 3$ Darthurs\%2Bveterinary\%2Breproductio n\%2Band\%2Bobstetrics\%2Bdavid\%2Be \%2Bnoakes\%26sortb y\%3D17\&cm_sp=snip pet-_-srp1-_-title9

[6] Robert SJ (2000). Veterinary Obstetrics and genital diseases. Theriogenology 5th ed. Edwards" Brothers, INC., Michigan, Pp. 48-104.

[7] Erb HN, Martin SW (1980). Interrelationships between production and reproductive diseases in Holstein cows. Age and seasonal patterns. J. Dairy Sci. 63 (11): 1918-1924.

[8] Juyal PD, Bal MS, Singla LD (2011) Economic impact, diagnostic investigations and management of protozoal abortions in farm animals. In: All India SMVS ${ }^{e e}$ Dairy Business Directory 11: 39-46.

[9] Msangi BSJ, Bryant MJ, Thorne PJ (2005). Some factors affecting variation in milk yield in crossbred dairy cows on smallholder farms in North-east Tanzania. Trop. Health Prod. 37 (5): 403-412.

[10] Bureau of Planning and Economic Development of Oromia Regional State (BPEDORS) (2000). Physical and SocioEconomical Profile of 180 District of Oromia Region. Bureau of Planning and Economic Development, Council of Regional State of Oromiya. Ethiopia. Available at: http://ibrary.ecsu.edu.et/cgibin/koha/opacdetail.pl?biblionumber $=13192$
[11] Central Statistical Authority (CSA) (2008/2009). Agricultural Sample Survey 2008/2009 [2001 E.C.]. Report on Livestock and Livestock Characteristics (Private Peasant Holdings), Addis Ababa, Ethiopia. P 120.

[12] Thrusfield M (2005). Veterinary Epidemiology. 3rded. Ames, Iowa: Blackwell Publishing. pp. 345-356.

[13] Nicholson MJ, Butterworth MH (1986). A guide to condition scoring of zebu cattle. International Livestock Ceneter for Africa, Addis Ababa, Ethiopia. pp. 1-5. Available at: http://www.delavidaboran.co.za/temp/article_A\%20Guide\%20to $\% 20 \mathrm{C}$ ondition $\% 20 \mathrm{Score} \% 20 \mathrm{of} \% 20 \mathrm{Zebu} \% \overline{2}$ CCattle $\% 20 \mathrm{Pg} 1$.pdf

[14] Bitew M, Prasad S (2011). Study on major reproductive health problems in indigenous and cross breed cows in and around Bedelle, South West Ethiopia. J. Anim. Vet. Adv. 10: 723-727.

[15] Gashaw A, Worku F, Mulugeta S (2011). Assessment of Small Holder Dairy Production System and Their Reproductive Health Problems in Jimma Town, Southwestern Ethiopia. Available at: http://www.jarvm.com/articles/Vo19Iss1/Vo19\%20Iss1Gashaw.pdf

[16] Hadush A, Abdella A and Regassa F (2013). Major pre-partum and postpartum reproductive problems of dairy cattle in Central Ethiopia. J. Vet. Med. Anim. Health 5 (4): 118-123.

[17] Temesgen A, Tegegn G (2015). Incidence of major clinical reproductive health problems of dairy cows at Bako livestock research farm over a two-year period (September 2008December 2010). Anim. Vet. Sci. 3 (6): 158-165.

[18] Mamo T (2004). "Study on major postpartum reproductive problems of smallholder dairy cows in and around DebreZeit. DVM Thesis, Faculty of Veterinary Medicine, Addis Ababa University, DebreZeit, Ethiopia," unpublished.

[19] Adane H, Yisehak T, Niguse T (2014). Assessment of major reproductive disorders of dairy cattle in Urban and Per-Urban area of Hosanna, Southern Ethiopia. J. Anim. Vet. Sci. 2 (5): 135-141.

[20] Tesfaye D, Shamble A (2013). Reproductive health problems of cows under different management systems in Kombolcha, Noetheast Ehiopia. Adv. Biomed. Res. 7: 104-108.

[21] Getachew E, Nibret M (2014). Department of veterinary clinical studies, faculty of veterinary medicine, University of Gondor, Gondor, Ethiopia. 13 (4): 444-449.

[22] Noakes DE (1986). Fertility and Obstetrics in Cattle Blackwell Scientific Publications. pp. 28-30.

[23] Kidusan K (2009). "Study on major reproductive disorders of dairy cattle in and around Wukro, DVM Thesis, College of Veterinary Medicine, Mekelle University, Tigray, Northern Ethiopia," unpublished.

[24] Molalegn and Shiv (2013): Study on Major Reproductive Health Problems in Indigenous and Cross breed cows in and around Bedelle, South West Ethiopia, Journl of Animal Health and Veterinary Advances 10 (6): 723-727.

[25] Benti AD, Zewdie W (2014). Major reproductive health problems of indigenous Borena cows in Ethiopia. J. Adv. Vet. Anim. Res. 1 (4): 182-188. 